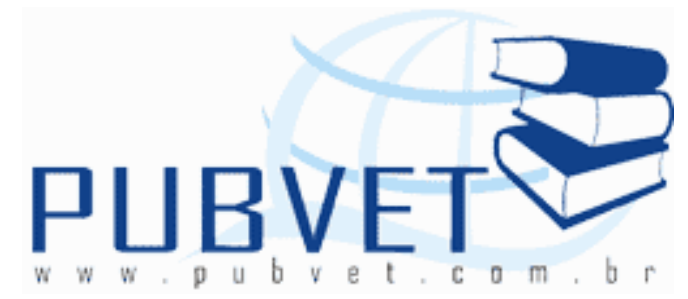

PUBVET, Publicações em Medicina Veterinária e Zootecnia.

\title{
Biotecnologias reprodutivas e fisiologia reprodutiva da fêmea bovina - conhecimento para o sucesso
}

\footnotetext{
Klayto José Gonçalves dos Santos ${ }^{1}$, Aracele Pinheiro Pales dos Santos ${ }^{1}$, Miliane Alves da Costa ${ }^{2}$ e Iván Salamanca Montesinos ${ }^{3}$

${ }^{1}$ Docentes da Universidade Estadual de Goiás, klayto.santos@ueg.br

${ }^{2}$ Bolsista PIBIC/UEG, graduanda em Zootecnia, Universidade Estadual de Goiás ${ }^{3}$ Doutorando em Ciência Animal, Universidade Federal de Goiás
}

\section{Resumo}

As biotecnologias buscam a eficiência reprodutiva dos rebanhos bovinos. Destas as mais utilizadas com sucesso são a inseminação Artificial (IA) e transferência de embriões (TE). Outras biotécnicas como a produção in vitro de embriões (PIVE) e clonagem, também contribuem para acelerar o melhoramento genético, porque incluem a utilização de animais superiores. Algumas biotecnologias utilizadas comercialmente em rebanhos leiteiros e de corte possuem limitações, dentre elas a PIVE, a qual sofre influência de vários fatores, como a taxa de recuperação oocitária e a variação individual na resposta ovariana aos tratamentos hormonais, requerendo mais estudos para avaliar o efeito da associação entre o início da onda folicular e o início do tratamento superestimulatório, tornando-se indispensável o amplo conhecimento da fisiologia reprodutiva da fêmea bovina.

Palavras-chave: embriões, onda folicular, oócitos. 


\title{
Biotechnology reproductive and physiology reproductive female bovine - knowledge for success
}

\begin{abstract}
The biotechnologies search the reproductive efficiency of the bovine herds. Of these the most used with success are the artificial insemination (IA) and embryo transfer (TE). Other biotechniques such as the in vitro embryo production (PIVE) and cloning, also contribute for accelerating the genetic improvement, because they include the use of superior animals. Some biotechnologies used commercially in dairy and beef herds have limitations, among them the PIVE, which suffer influence of several factors, like the oocyte recovery rate and the individual variation in the ovarian response to hormonal treatments, requiring more studies for evaluating the effect of the association between the initiation of the follicular wave and the initiation of the superstimulatory treatment, becoming indispensable the ample knowledge of the reproductive physiology of the bovine female.
\end{abstract}

Keywords: embryos, follicular wave, oocytes.

\section{INTRODUÇÃO}

Os índices reprodutivos e produtivos da bovinocultura brasileira estão muito abaixo do desejável, e a elevação na produção de leite nas últimas décadas ocorreu, em parte, muito mais pela expansão das áreas exploradas e o aumento do efetivo de rebanho que por um real incremento na produtividade. Quanto mais maximizada for a produção, utilizando biotecnologias como inseminação artificial, sincronização estral, transferência de embriões e fecundação in vitro, tanto maior será a exigência para a eficiência produtiva ótima (NEVES et al., 2002).

Durante os últimos anos, a pecuária no Brasil sofreu um enorme ganho de volume e produtividade. A aplicação de técnicas modernas na produção, incluindo as biotecnologias ligadas à reprodução animal, contribuiu para que o 
país fosse classificado como o maior detentor do rebanho comercial de bovinos do mundo, o maior exportador mundial de carne bovina (SERAPIÃO, 2008) e, ainda o primeiro país do mundo em número de embriões bovinos produzidos in vitro (PAVÃO, 2009).

Do ponto de vista econômico, o desempenho reprodutivo é um dos principais focos da pecuária. Assim, a utilização de biotecnologias da reprodução é alternativa para aumentar os índices reprodutivos de um rebanho bovino (RUBIN, 2006).

O desenvolvimento e aplicação de biotécnicas reprodutivas são condições indispensáveis para a melhoria na eficiência reprodutiva, e especialmente para os ruminantes domésticos. Técnicas como a inseminação artificial (IA) e transferência de embriões (TE) tem sido utilizadas com sucesso. Outras biotécnicas como a produção in vitro de embriões, clonagem e transgenia, contribuem para acelerar o melhoramento genético, pois incluem a utilização de animais provados e com características superiores desejáveis, mas apresentam ainda resultados inferiores em termos de produção comercial.

Segundo THIBIER (2007), mais de 670.000 embriões produzidos in vivo foram transferidos em todo o mundo, dos quais aproximadamente a metade a fresco e a metade congelados. Em contrapartida, cerca de 292.000 embriões produzidos in vitro (PIV) foram transferidos no mesmo ano, dos quais cerca de $75 \%$ foi a fresco. O fato da fêmea Bos taurus indicus produzir dezenas de oócitos em um único procedimento de aspiração folicular, numa relação direta com o número de folículos (PONTES et al., 2009) elevou o Brasil à liderança mundial na produção in vitro de embriões. Adicionalmente, com o advento de novas tecnologias para a utilização de fármacos na área reprodutiva, mais especificamente os hormônios, é possível incrementar os índices reprodutivos dos rebanhos de corte e leite utilizando a hormonioterapia para programas de inseminação artificial e superestimulação ovariana em animais de elevado valor genético (KOZICKI et al., 2005).

Dentre as biotecnologias utilizadas, a transferência de embriões (TE) e a fertilização in vitro (FIV), possibilitam o aumento dos índices reprodutivos em 
fêmeas, intensificando a seleção de animais. A utilização dessas biotecnologias e seu desenvolvimento são essenciais para aumentar a eficiência produtiva dos rebanhos (REICHENBACH et al., 2002).

A TE consiste em obter embriões de uma fêmea doadora e transferí-los para fêmeas receptoras com a finalidade de completar o período de gestação. Sua importância básica para a produção animal está na possibilidade de uma fêmea produzir um número de descendentes muito superior ao que seria possível fisiologicamente, durante sua vida reprodutiva (REICHENBACH et al., 2002). A TE oferece uma série de vantagens para a seleção zootécnica com conseqüente reflexo sobre a produção animal, tais como, seleção de mães dos touros para inseminação, aumento do número de descendentes de animais geneticamente superiores, redução do intervalo entre gerações e aumento da velocidade do melhoramento (ANDRADE et al., 2002).

A principal meta do programa de TE é a superovulação, cujo objetivo é obter, após administração de hormônios exógenos, elevado número de embriões viáveis por doadora pelo aumento do número de oócitos liberados, possibilitando a transferência dos embriões obtidos, após a inseminação artificial no trato reprodutivo de receptoras e assim completarem a gestação (RUMPF et al., 2000).

Segundo DODE (2006), as fêmeas bovinas nascem com mais de 100.000 oócitos nos folículos ovarianos, mas pelas vias naturais, apenas $0,01 \%$ de produtos viáveis podem ser gerados, ou seja, um número próximo a dez descendentes em toda sua vida reprodutiva.

Uma das soluções propostas para esse problema foi o desenvolvimento da produção in vitro (PIV) de embriões bovinos, uma importante biotécnica de reprodução assistida, a qual permite a interação do espermatozóide com o oócito fora do trato reprodutivo feminino, com a formação de um novo indivíduo. O processo envolve as etapas de colheita, maturação in vitro (MIV) e fertilização in vitro (FIV) de oócitos, assim como cultivo in vitro (CIV) de zigotos e estruturas embrionárias fora do útero animal, ao contrário da clássica transferência de embriões (GONÇALVES et al., 2002). 
A técnica de produção in vitro tem sido utilizada nos diferentes segmentos da reprodução assistida humana e animal. Inicialmente essas técnicas foram empregadas somente na pesquisa fundamental como instrumento para estudar fenômenos fisiológicos relacionados aos gametas femininos e masculinos, permitindo assim o maior entendimento das fases de crescimento, maturação e fecundação de oócitos, além da capacitação espermática e desenvolvimento embrionário precoce (CALADO et al., 2001).

Embora essas biotécnicas sejam utilizadas em muitos rebanhos tanto de leite quanto de corte, a eficiência de um programa de PIV está limitada, entre outros fatores, pela taxa de recuperação de oócitos, pela variação individual na resposta ovariana, aos tratamentos que estimulam $\mathrm{o}$ amadurecimento e ovulação de um número grande de folículos. Requerendo-se estudos para avaliar o efeito da associação entre início da onda folicular e o início do tratamento superestimulatório, principalmente em animais de origem leiteira.

\section{REVISÃO DE LITERATURA}

\subsection{Estrutura ovariana}

Os ovários são órgãos pares do sistema reprodutor feminino, com localização e tamanho variado entre as espécies (ALVES et al., 2008), tendo nos bovinos, em média de 3,0 a 4,5 cm de comprimento e 1,5 a 2,0 cm de largura, com coloração rósea clara (JUNQUEIRA \& CARNEIRO, 1995).

Segundo MARIANI (2009), os ovários são os órgãos primários para a reprodução na fêmea, exatamente como os testículos no macho, e podem ser considerados de natureza endócrina e citogênica (produtora de células), já que produzem hormônios que são absorvidos diretamente pela corrente sanguínea, e também óvulos que são expelidos por estas glândulas.

O folículo, unidade morfofuncional do ovário, é uma estrutura altamente organizada e constituída basicamente pelos oócitos. Sua função é proporcionar 
um ambiente ideal para o crescimento, maturação oocitária e produção de hormônios (ALVES et al., 2008).

Segundo GONÇALVES et al. (2002), o oócito no interior do folículo está rodeado de células da granulosa, formando o complexo cumulus oócitos (CCO). O conjunto de células próximas da zona pelúcida (ZP), que estão em íntimo contato com o oócito por junções intercomunicantes é denominado corona radiata, e está circundado por células foliculares assim como demarcado por uma membrana basal que o separa do estroma ovariano.

A população folicular ovariana é bastante heterogênea. De acordo com os aspectos morfológicos, os folículos podem ser divididos em: 1) folículos préantrais ou não cavitários, que abrangem os folículos primordiais, primários e secundários e 2) folículos antrais ou cavitários, compreendendo os folículos terciários (subordinados e dominantes), de Graaf ou pré-ovulatório (MARTINS et al., 2008).

Os folículos primordiais se encontram em estádio de repouso e são compostos de um oócito imaturo circundado por uma única camada de células somáticas (pré-granulosa) de forma achatada. Os folículos primários são constituídos de um oócito em crescimento circundado por uma camada de células da granulosa de formato cubóide, não possuindo células tecais diferenciadas e podendo apresentar uma ZP em formação. Os folículos secundários são caracterizados por um oócito inteiramente circundado por uma $Z P$, e a presença de pelo menos duas camadas de células da granulosa de forma cubóide. Ao contrário dos folículos primordiais, os folículos primários e secundários são considerados folículos em estádio inicial de crescimento (MARIANI, 2009).

Os folículos terciários são constituídos por um oócito circundado pela corona radiata e células do cumulus que conectam o oócito às células da granulosa, além das células tecais e uma cavidade contendo líquido folicular. Os folículos de Graaf ou pré-ovulatórios apresentam todos os componentes presentes nos folículos terciários, contudo o oócito apresentar-se-á no estádio final do desenvolvimento. De toda a população folicular presente no ovário, 
apenas cerca de $0,01 \%$ destes atingirá a ovulação, enquanto os demais folículos sofrem um processo degenerativo ou apoptótico conhecido por atresia, durante o desenvolvimento folicular (MAX et al., 2004).

\subsection{Ciclo estral}

A fisiologia do ciclo estral é complexa e depende da perfeita interação entre o sistema nervoso central, sistema endócrino e os órgãos genitais. 0 hipotálamo, estrutura localizada centralmente na base do cérebro possui núcleos responsáveis pela secreção de hormônios reguladores de gonadotrofinas (GnRH), os quais são liberados de forma pulsátil ligando-se, posteriormente aos receptores da hipófise. Por conseguinte dá-se início a síntese e liberação, também pulsátil, dos hormônios de natureza glicoprotéica, denominados hormônio folículo estimulante (FSH) e hormônio luteinizante (LH) (MORAES et al., 2002; JAINUDEEN \& HAFEZ, 2004). O FSH secretado pelo lobo anterior da hipófise promove crescimento folicular e hiperplasia nas células da granulosa e teca interna nos ovários, enquanto o LH induz a maturação e ovulação dos folículos, produção de estrógenos pela teca interna e luteinização desta e da granulosa com desenvolvimento do corpo lúteo (GRUNERT \& GREGORY, 1989).

O ciclo estral em bovinos apresenta duração média de 21 dias e é definido como o intervalo transcorrido entre dois estros, no qual ocorre uma série de alterações hormonais, morfológicas e comportamentais (MOREIRA et al., 2000; ALBUQUERQUE et al., 2004; JAINUDEEN \& HAFEZ, 2004). Pode ser dividido em duas fases distintas: a fase folicular ou estrogênica caracterizada pelo pró-estro e estro culminando com a ovulação, e a fase luteínica ou progesterônica constituída pelo metaestro e diestro finalizando-se com a luteólise (MACMILLAN \& BURKE, 1996; MORAES et al., 2002; ALBUQUERQUE et al., 2004). Segundo ALBUQUERQUE et al. (2004), o estro é comumente denominado de dia zero do ciclo estral. Dura entre 12 e 24 horas na vaca, variando entre as raças e inicia em sincronia ao pico de $\mathrm{LH}$, resultando em dois 
fenômenos independentes: a luteinização das células da granulosa e teça, e a ruptura do folículo ovulatório. Em seguida ocorre a ovulação e posterior formação do corpo lúteo (MORAES et al., 2002). JAINUDEEN \& HAFEZ (2004) descreveram que, no ovário, esse período do ciclo estral é caracterizado pela elevada secreção de estrógeno (E2) dos folículos pré-ovulatórios.

De acordo com MORAES et al. (2002), os sinais de estro ocorrem logo antes da ovulação, devido ao aumento de estradiol na circulação sanguínea. Os elevados níveis de estrógeno, na fase inicial do estro, sinalizam o hipotálamo para inibir o feedback negativo estimulando a liberação cíclica de GnRH (feedback positivo) (ALBUQUERQUE et al., 2004). Nessa fase a fêmea apresenta vulva edemaciada, mucosa vaginal hiperêmica, descarga de muco vaginal claro e elástico, inserção da cauda arrepiada, lordose, inquietude, há formação de grupo de animais e finalmente, quando montada, fica imóvel. Para MORAES et al. (2002) e ALBUQUERQUe et al. (2004), o sinal mais característico e confiável é quando a fêmea aceita a monta sem reagir.

\subsubsection{Fase folicular ou estrogênica}

Durante o ciclo estral, o crescimento dos folículos ovarianos, em bovinos, ocorre em um padrão denominado ondas de crescimento folicular.

Normalmente há a emergência de duas ou três ondas de crescimento folicular (ADAMS et al., 1992; BO et al., 1994; BINELLI et al., 2001). De acordo com MORAES et al. (2002) e ALBUQUERQUE et al. (2004), este crescimento folicular ocorre sob baixas concentrações plasmática de progesterona (P4) e alta pulsatibilidade de LH. Uma onda de folículos emerge entre os dias um e três após o estro. São geralmente em torno de 10 a 50 folículos neste grupo com um tamanho de dois a três milímetros de diâmetro. Nos dias subseqüentes parte desses folículos cresce para quatro a seis milímetros, sendo que dois a cinco folículos maiores do grupo continuarão a crescer, enquanto outros regridem (MOREIRA et al., 2000). Para DISKIN et al. 
(2002), cada onda de crescimento folicular divide-se na fase de recrutamento, seleção, dominância e atresia ou ovulação.

No momento em que ocorre a lise do corpo lúteo $(\mathrm{CL})$, as concentrações de progesterona declinam favorecendo o aumento transitório das concentrações plasmáticas de $\mathrm{FSH}$, caracterizando assim a fase de recrutamento ou emergência (ADAMS et al., 1992). Conseqüentemente, ocorre o crescimento do folículo (MORAES et al., 2002; ALBUQUERQUE et al., 2004) até em torno do oitavo dia após o estro, com emergência da segunda onda de crescimento folicular reiniciando o processo (BO et al., 1994; MOREIRA et al., 2000). A taxa de seleção é semelhante para todos os folículos recrutados, no entanto, na fase de seleção um folículo passa a apresentar maior taxa de crescimento e torna-se dominante e outros entram em atresia (GINTHER et al., 2001; DISKIN et al., 2002). A fase de regressão ocorre após o décimo dia, para fêmeas que apresentam duas ondas de crescimento folicular, sendo que na existência de três ondas têm-se do sétimo ao oitavo dia para regressão (SILCOX et al., 1993; BO et al., 1994).

\subsubsection{Fase luteínica ou progesterônica}

A fase luteínica caracteriza-se pela formação do $C L$ ovariano, resultante do rompimento de um folículo ovulatório e presença de maiores concentrações plasmáticas de progesterona. Condição esta, que leva ao crescimento e atresia dos folículos ovarianos devido à diminuição da pulsatibilidade e ausência do pico de LH, ou seja, pulsos de LH com alta frequência e baixa amplitude (MORAES et al., 2002; ALBUQUERQUE et al., 2004).

Conforme BINELLI et al. (2001); MORAES et al. (2002); ALBUQUERQUE et al. (2004) e JAINUDEEN \& HAFEZ (2004) à medida que vai ocorrendo a luteinização das células foliculares e formação do corpo lúteo, as estruturas foliculares, que até então secretavam estrógeno, passam a secretar progesterona, hormônio essencial para a ciclicidade normal da fêmea bovina (BO et al., 2002). Segundo ALBUQUERQUE et al. (2004) nesta fase a fêmea 
não aceita mais a monta, o cérvix apresenta-se fechado, o muco produzido é viscoso e não flui mais pela comissura vulvar. Os autores ainda acrescentam que no primeiro e segundo dia do metaestro podem ocorrer sangramentos uterinos, que se atribui à queda nos níveis de estrógeno. Para BO et al. (2002), durante a fase de secreção de progesterona ocorrem aumentos periódicos de $\mathrm{FSH}$, o qual estimula a emergência de ondas foliculares.

O diestro, que começa no dia seis do ciclo estral, dura entre dez e 14 dias, sendo a fase mais longa do ciclo na vaca. Nesta fase o corpo lúteo continua a se desenvolver atingindo o máximo de seu crescimento e produção de progesterona, que impede a onda gonadotrófica de completar a maturação de folículos presentes no ovário, em função do crescimento folicular pósovulatório, sobrevindo a atresia dos mesmos. O útero continua com sua musculatura relaxada, o endométrio mostra-se espessado com glândulas hipertrofiadas. O cérvix está fechado com muco denso e viscoso, a vagina apresenta-se com as mucosas pálidas e secas (ALBUQUERQUE et al., 2004).

Caso o concepto esteja presente entre os dias 14 e 17 e ocorra secreção adequada de interferon, não ocorrerá a liberação de prostaglandinas (PGF2a) e a progesterona continuará a ser secretada para manter a gestação (ALBUQUERQUE et al., 2004; JAINUDEEN \& HAFEZ, 2004). Caso não haja fecundação ou a produção de interferon for comprometida nesse período, a PGF2a será liberada com conseqüente luteólise, liberação e queda dos níveis plasmáticos de progesterona, proporcionando condições favoráveis para um novo crescimento folicular e ovulação (OKUDA et al., 2002).

O entendimento dos mecanismos básicos sobre a fisiologia da reprodução e interação com outros sistemas fornecem embasamento para discutir e aplicar as diferentes biotécnicas reprodutivos, destacando a TE.

\subsection{Foliculogênese}

A função primária de um folículo ovariano de mamíferos é a liberação de um oócito apto para ser fertilizado. A foliculogênese pode ser definida como 
a formação de folículos maduros, pré-ovulatórios, a partir de uma reserva de folículos primordiais (SPICER \& ECHTERNKAMP, 1986). Este processo consiste no crescimento do oócito, diferenciação de uma camada de células granulosas (planas), e de uma camada celular (teca interna) para fora da membrana basal (GREENWALD \& TERRANOVA, 1988).

Da reserva de folículos primordiais, formada durante a vida fetal ou após nascimento, alguns folículos crescem continuamente durante a vida do animal, ou até a reserva se exaurir. Quando um determinado folículo deixa essa reserva, ele cresce até a ovulação ou até que ocorra a sua degeneração, o que acontece com a maioria dos folículos (HAFEZ \& HAFEZ, 2004).

$O$ início e a regulação do desenvolvimento folicular pré-antral são predominantemente conduzidos por fatores produzidos localmente (McNATTY et al., 1999). O oócito tem um papel ativo na coordenação da proliferação e diferenciação das células da granulosa ao seu redor (GILCHRIST, 2004). A comunicação intercelular é proporcionada por processos citoplasmáticos transzonais (TZP), que são extensões das células da granulosa que penetram através da zona pelúcida e atingem a membrana do oócito. Este tipo de comunicação, entre o oócito e células somáticas, parece ser regulada durante o desenvolvimento, uma vez que às TZPs retraem quando o folículo atinge o estágio pré-antral, que se acredita efeito do FSH (ALBERTINI et al, 2001).

O passo seguinte na foliculogênese é a formação do antro, por meio da coalescência de pequenas gotas de fluido folicular, secretadas pelas células da granulosa. O número de folículos antrais ou terciários depende do nível de gonadotrofinas. Uma percentagem inferior a $1 \%$ dos folículos se desenvolverá até a fase pré-ovulatória, sendo que os outros sofrerão um processo de atresia ou regressão (RIVERA, 1993).

\subsubsection{Recrutamento e seleção de folículos ovarianos}

O folículo ovariano é uma unidade fisiológica equilibrada cujas estruturas e funções dependem de fatores extracelulares, como as 
gonadotrofinas e um complexo sistema de interações intrafoliculares (HAFEZ \& HAFEZ, 2004).

O fluido folicular possui constituintes específicos, como esteróides, e glicoproteínas sintetizadas pelas células da parede folicular. Em folículos antrais grandes, o fluido folicular contém concentrações marcadamente elevadas de $17 \beta$ estradiol na fase folicular, e progesterona com a aproximação da ovulação (HAFEZ \& HAFEZ, 2004).

Uma elevação nas concentrações plasmáticas de FSH estimula o recrutamento folicular e a emergência da onda folicular (ADAMS et al., 1992; FORTUNE et al., 2004). Em espécies monovulatórias, ou seja, que ovulam apenas um oócito, um folículo é selecionado do grupo de recrutados e adquire capacidade ovulatória, enquanto os folículos subordinados entram em atresia. O folículo selecionado é conhecido como folículo dominante e desempenha um papel ativo na supressão do crescimento dos subordinados pela secreção de estradiol e inibina (GINTHER et al., 1996). Em bovinos os folículos podem atingir o diâmetro de $8 \mathrm{~mm}$ independentemente do suporte de $\mathrm{LH}$, mas o crescimento além de $9 \mathrm{~mm}$ requer LH endógeno ou FSH exógeno (GONG et al., 1996). A aquisição de receptores de LH pelas células da granulosa é essencial para a maturação folicular.

A partir dos primeiros estudos ultrasonográficos, que datam de meados da década de 80 , este desenvolvimento sincrônico de grupos de folículos em determinados períodos do ciclo estral é denominado de onda folicular (PIERSON \& GINTHER, 1984). Segundo KNOPF et al. (1989), tem sido demonstrado que mais de $95 \%$ dos ciclos estrais são constituídos de duas ou três ondas foliculares. Essas variações no número de ondas de cada ciclo podem ocorrer em função de vários fatores, como dieta, manejo, fotoperíodo, pós-parto imediato, etc. (GINTHER et al., 1996).

Em um determinado momento do ciclo, ocorre o recrutamento de um grupo de folículos primordiais para iniciar o crescimento (fase de recrutamento), dentre os recrutados um é selecionado (fase de seleção), este se destaca e cresce mais rapidamente que os outros (fase de dominância), 
com a conseqüente atresia dos demais folículos da onda. Uma nova onda se inicia somente quando o folículo dominante da onda anterior ovula ou inicia o processo de atresia. O folículo dominante entra em atresia, quando há altos níveis de progesterona (diestro ou gestação) (HAFEZ \& HAFEZ, 2004).

A determinação do número de ondas por ciclo vai influenciar no tratamento superovulatório, que deve começar no início de uma onda de desenvolvimento folicular, antes do estabelecimento da dominância, para que forneça resultados satisfatórios (BARUSELLI et al., 2004).

\subsection{Dinâmica folicular}

Apesar da enorme quantidade de informações produzidas durante as últimas décadas, o entendimento completo dos mecanismos controladores do desenvolvimento folicular, ainda não foi atingido. A regulação do desenvolvimento folicular é complexa e envolvem fatores endócrinos, parácrinos e autócrinos, que são orquestrados de maneira estágio-específica a fim de controlar vários processos incluindo proliferação e diferenciação de células foliculares, esteroidogênese, angiogênese/vascularização, remodelagem da membrana basal e matriz extracelular, assim como atresia/apoptose (WEBB et al., 2003; ACOSTA \& MIYAMOTO, 2004; FORTUNE et al., 2004).

$\mathrm{O}$ ovário tem duas funções principais. A primeira é a produção cíclica de oócitos fertilizáveis. A segunda é a produção de hormônios esteróides, em proporções balanceadas. O folículo é a estrutura ovariana que permite ao ovário desenvolver suas duas funções: a gametogênese e a esteroidogênese (HAFEZ \& HAFEZ, 2004).

Segundo MACMILLAN \& THATCHER (1991) e FIGUEIREDO et al. (1997), conhecendo a dinâmica folicular, pode-se aumentar a eficiência reprodutiva com a utilização de fármacos, principalmente, em programas de inseminação artificial ou de transferência de embriões. 


\subsubsection{Controle da dinâmica folicular}

Nos últimos dez anos foram desenvolvidos alguns métodos para controlar o início da onda de crescimento folicular em bovinos. Entre estes se pode citar métodos mecânicos, como remoção física do folículo dominante (BERGFELT et al., 1994; MARTINEZ et al., 2000) e métodos farmacológicos como o GnRH (PURSLEY et al., 1995) ou a associação de estrógeno e progesterona/progestágenos (BO et al., 1993, 1995).

Estes métodos têm sido empregados com êxito para promover o início da onda de crescimento folicular em intervalo de tempo conhecido.

Em novilhas Bos taurus taurus $x$ Bos taurus indicus, BARUSELLI et al. (2003) observaram que a aplicação de GnRH em momentos aleatórios do ciclo estral induz a ovulação em apenas 45,7\% (16/35) das fêmeas. Em outro trabalho BARUSELLI et al. (2004), colocaram em questão o uso do GnRH em novilhas Bos taurus indicus, justamente por apresentarem baixas taxas de ovulação.

Em Bos taurus indicus, o tratamento eletivo para a indução da emergência da nova onda de crescimento folicular é a associação de estradiol $\left(E_{2}\right)$ e progesterona $\left(P_{4}\right)$. A eficiência desta associação tem sido descrita em diversos trabalhos em fêmeas Bos taurus taurus (BO et al., 1993, 1994, 1995; MARTINEZ et al., 2000; COLAZO et al., 2003, 2005). CARVALHO (2004) estudou o efeito do tratamento com estradiol e progesterona na emergência da onda folicular em novilhas Bos taurus indicus e Bos taurus taurus mantidas nas mesmas condições de manejo. Não se observou diferença no intervalo entre o tratamento com benzoato de estradiol e a emergência folicular entre, Bos taurus indicus e Bos taurus taurus. Entretanto, novilhas Bos taurus indicus apresentaram maior número de folículos recrutados no início da onda de crescimento folicular que novilhas Bos taurus taurus, sugerindo maior resposta super estimulatória ovariana ao tratamento com gonadotrofina.

Diferentes tipos de ésteres de estradiol, incluindo o Benzoato de estradiol (BE), Valerato de estradiol (VE) e Cipionato de estradiol (CE) estão 
disponíveis comercialmente na América do Sul. Todos estes ésteres são capazes de induzir a regressão de folículos antrais quando administrados na presença de elevadas concentrações de progesterona (BO et al., 1995). O Valerato de estradiol e o Cipionato de estradiol apresentam meia vida mais longa, resultando na indução de uma nova onda folicular mais tardia e menos sincronizada (BO et al., 1995; COLAZO et al., 2003; COLAZO et al., 2005) que o $17 \beta$ estradiol (BO et al., 1995) ou Benzoato de estradiol (BO et al., 2002).

SÁ FILHO et al. (2005) avaliaram a administração de $2 \mathrm{mg}$ de BE no momento da inserção do implante auricular contendo $3 \mathrm{mg}$ de Norgestomet (Crestar ${ }^{\circledR}$, Intervet, Brasil) ou do dispositivo intravaginal de progesterona $\left(\right.$ CIDR $^{\circledR}$; Pfizer, Brasil) em novilhas Bos taurus indicus. O intervalo entre o tratamento e a emergência da nova onda folicular não foi influenciado pelo tratamento com $\operatorname{CRESTAR}^{\circledR}(2,9 \pm 0,1$ dias $)$ ou $\operatorname{com} \operatorname{CIDR}(3,1 \pm 0,1$ dias $)$. Em outro recente estudo SÁ FILHO et al. (2006) avaliaram o efeito do BE ou de duas diferentes doses de VE no momento e na sincronização da emergência da onda folicular em vacas e novilhas Bos taurus indicus tratadas com implante auricular de Norgestomet ${ }^{\circledR}$. Foi observado efeito significativo de tratamento, no entanto, não foi observada interação entre tratamento e categoria animal (vaca ou novilha) para o intervalo e dispersão do momento da indução da emergência da nova onda de crescimento folicular.

Estudos recentes avaliaram a necessidade do uso de progesterona injetável no início do tratamento com dispositivo intravaginal de progesterona (DIB) em vacas Nelore (Bos taurus indicus). MARTINS et al. (2005) observaram maior sincronização na emergência e atraso no dia de início da onda de crescimento folicular quando se utilizou a associação progesterona injetável mais $B E(4,2 \mathrm{mg})$ que em animais que receberam somente $B E$ no início do tratamento com dispositivos intravaginais novos $(3,1 \mathrm{mg})$ ou reutilizados (2,8 $\mathrm{mg}$ usados uma vez), concordando com o descrito para fêmeas Bos taurus taurus (MORENO et al., 2001). Em outro estudo, SÁ FILHO et al. (2005) testaram o efeito de diferentes doses (2 ou $3 \mathrm{mg}$ ) e/ou momentos (Dia 0 ou Dia 1) da aplicação de BE ou a associação de BE mais 
progesterona injetável no início do tratamento com dispositivo intravaginal de progesterona (Dia 0) em vacas Nelore. O tratamento com BE+P4 no Dia 0, apresentou melhor sincronização da emergência da onda de crescimento folicular $(4,0 \mathrm{mg})$ que os tratamentos com apenas BE (2mg no D0). Portanto, pode-se concluir que o tratamento com Benzoato de estradiol associado à progesterona injetável no início do tratamento promove menor dispersão do momento do início da emergência da nova onda folicular.

\subsubsection{Fatores que afetam a reposta superestimulatória}

A variabilidade na resposta das doadoras aos tratamentos superovulatórios com as gonadotropinas continua sendo um dos maiores problemas em programas comerciais de TE (MAPLETOF et al., 2002; NOGUEIRA et al., 2002, BARROS et al., 2004). Esta variação individual ao tratamento superovulatório foi também relatada em vacas Nelore (Bos taurus indicus) utilizando delineamento experimental "cross over" (BARUSSELI et al., 2003). Os autores descrevem efeito significativo $(p<0,05)$ de doadora no número de corpos lúteos (CLs) no dia da colheita, na taxa de recuperação, no total de estruturas recuperadas, assim como no número de embriões transferíveis e congeláveis, indicando que a doadora foi a principal fonte de variação dos resultados.

DONALDSON (1992) demonstrou que apesar do cumprimento de todos os requisitos, a resposta superovulatória resulta em muitas variações. Um estudo que envolveu 1263 doadoras mostrou que somente 68 \% das fêmeas induzidas a superovulação produziram embriões transferíveis. A superovulação é a etapa menos previsível dentro da técnica de transferência de embriões, sendo vários os fatores que influenciam os resultados, e mesmo quando praticamente não ocorreu nenhuma alteração de uma superovulação para outra, em um mesmo animal, os resultados podem ser diferentes.

A resposta ovariana também está relacionada aos diferentes protocolos superovulatórios, bem como o tipo, partida ou dose total de gonadotrofina e 
do momento do início do tratamento. Existem outros importantes fatores que podem influenciar a resposta superovulatória, tais como, a condição nutricional, idade, histórico reprodutivo, estação do ano e condição ovariana no momento do início do tratamento (KAWAMATA, 1994; YAAKUB et al., 1999; MAPLETOFT et al., 2002).

No protocolo tradicional de superestimulação ovariana, o tratamento com gonadotrofinas é iniciado na metade do ciclo estral (oito e doze dias após ovulação). Esta metodologia apresenta algumas dificuldades por requerer a detecção do "cio base" para o início do tratamento superovulatório. Essa característica pode afetar a eficiência dos protocolos de superestimulação ovariana (MAPLETOFT et al., 2002).

Diversos estudos têm demonstrado a importância do momento do início do tratamento superovulatório e o início da onda de crescimento folicular. A ausência do folículo dominante no início do tratamento com gonadotrofinas aumenta a eficiência dos programas de superestimulação ovariana (ADAMS, 1994; MAPLETOFT et al., 2002). NASSER et al. (1993) obtiveram maior número de folículos recrutados e maior taxa de ovulação quando o tratamento superovulatório foi realizado um dia antes ou no dia de início da emergência da onda de crescimento folicular que os tratamentos realizados um ou dois dias após o início da onda.

Sendo assim, alternativas para o controle da emergência da onda de crescimento folicular em momentos aleatórios do ciclo estral, sem a necessidade de detecção do estro para o estabelecimento do "cio base", podem facilitar o manejo de doadoras, bem como possivelmente aumentar a eficiência dos programas de transferência de embriões em rebanhos zebuínos e taurinos.

\section{CONSIDERAÇÕES FINAIS}

Diversos estudos estão sendo realizados para aperfeiçoar as biotecnologias reprodutivas. Mas para que os resultados sejam alcançados, necessita de melhor conhecimento da fisiologia reprodutiva das fêmeas bovinas, melhoria dos sistemas de produção e da qualidade do rebanho. 
As biotecnologias reprodutivas são alternativas para aumentar a quantidade de animais superiores na raça, essas ferramentas vêm contribuindo para que o país aumente anualmente sua produção e sendo classificado como um dos maiores emergentes em produção de carne e leite no mundo.

A viabilidade econômica das biotecnologias está intimamente relacionada à eficiência dos laboratórios. Não apenas com relação à taxa de produção dos embriões, mas principalmente com a qualidade do embrião, a capacidade de estabelecer gestação, desenvolvimento fetal e placentário normais, obtenção de gestações a termo e nascimento de crias saudáveis e viáveis.

O sucesso das biotecnologias reprodutivas depende de um controle de qualidade eficiente, de um profissional qualificado, com conhecimento amplo da fisiologia reprodutiva bovina e da utilização correta em dias e horas de todos os procedimentos.

É importante ressaltar que as biotecnologias reprodutivas não é a solução para os problemas reprodutivos nas propriedades, mas se o sistema estiver funcionando satisfatoriamente, é uma ferramenta fundamental para alcançar, ou mesmo, melhorar o potencial genético das fêmeas bovinas.

\section{REFERÊNCIAS}

ACOSTA, T. J.; MIYAMOTO, A. Vascular control of ovarian function: ovulation, corpus luteum formation and regression. Animal Reproduction Science, v. 82-83, p. 127-140, 2004.

ADAMS, G. P.; MATTERI, R. L.; KASTELIC, J. P.; KO, J. C.; GINTHER, O. J. Association between surges of follicle-stimulating hormone and the emergence of follicular waves in heifers. Journal of Reproduction and Fertility, v. 94, p. 177-188, 1992.

ADAMS, G. P. Control of ovarian follicular wave dynamics in cattle: Implications for synchronization \& superstimulation. Theriogenology, v. 41, p. 19-24, 1994.

ALBERTINI, D. F.; COMBELlES, C. M.; BENECCHI, E.; CARABATSOS, M. J. Cellular basis for paracrine regulation of ovarian follicle development. Reproduction, v. 121, p. 647-653, 2001.

ALBUQUERQUE, F. T.; FILHO, J. B. B.; VIANA, J. H. M. Manipulação do ciclo estral em bovinos de corte: bases anatômicas, fisiológicas e histológicas da reprodução da fêmea. Lavras (MG): UFL - Departamento de Medicina Veterinária, 2004. 
ALVES, B. G; NEVES, S. M. N; ARRUDA, R. P; NAVES, J. H. F. F; ALVES, K. A. A classificação do corpo lúteo por assimetria ovariana e sua relação com os índices de prenhes em receptoras de embriões bovinos. In: XXXV Congresso Brasileiro de Medicina Veterinária CONBRAVET. Anais..., Gramados- RS, 2008.

ANDRADE, J. C. O.; OLIVEIRA, M. A. L.; LIMA, P. F. Use of steroid hormone treatments prior to superovulation in Nelore donors. Animal Reproduction Science, v. 69, n.1-2, p. 9-14, 2002.

BARROS, C. M.; NOGUEIRA, M. F. Embryo transfer in Bos indicus cattle. Theriogenology, v. 56, p. $1483-1496,2001$.

BARUSELLI, P. S.; MARQUES, M. O.; REIS, E. L.; NASSER, L. F. T.; SILVA, R. C. P.; MENEGATTI, J. A.; VALENTIN, R.; SANTOS, I. C. C. Adequação da dose de FSH (Folltropin-v) em protocolos de superovulação de vacas Nelore (Bos taurus indicus) com inseminação artificial em tempo fixo (SOTF). Acta Scientiae Veterinarie, v. 31, p. 244-245, 2003.

BARUSELLI, P. S.; REIS, E. L.; MARQUES, M. O.; NASSER, L. F.; BO, G. A. The use of hormonal treatments to improve reproductive performance of anestrous beef cattle in tropical climates. Animal Reproduction Science, v. 82-83, p. 479-86, 2004.

BERGFELT, D. R.; LIGHTFOOT, K. C.; ADAMS, G. P. Ovarian synchronization following ultrasound-guided transvaginal follicle ablation in heifers. Theriogenology, v. 42, p. 895-907, 1994.

BINELLI, M.; THATCHER, W. W.; MATTOS, R.; BARUSELLI, P. S. Antiluteolytic strategies to improve fertility in cattle. Theriogenology, v. 56, p. 1451-1463, 2001.

BO, G. A.; ADAMS, G. P.; NASSER, L. F.; PIERSON, R. A.; MAPLETOFT, R. J. Effect of estradiol valerate on ovarian follicles, emergence of follicular waves and circulating gonadotropins in heifers. Theriogenology, v. 40, n. 2, p. 225-239, 1993.

BO, G. A.; ADAMS, G. P.; PIERSON, R. A.; TRÍBULO, H. E.; CACCIA, M.; MAPLETOFT, R. J. Follicular wave dynamics after estradiol-17 $\beta$ treatment of heifers with or without a progestogen implant. Theriogenology, v. 41, n. 8, p. 1555-1569, 1994.

BO, G. A.; ADAMS, G. P.; CACCIA, M.; MARTINEZ, M.; PIERSON, R. A.; MAPLETOFT, R. J. Ovarian follicular wave emergence after treatment with progestogen and estradiol in cattle. Animal Reproduction Science, v. 39, p. 193-204, 1995.

BO, G. A.; BARUSELLI, P. S.; MORENO, D.; CUTAIA, L.; CACCIA, M.; TRÍBULO, R.; TRÍBULO, H.; MAPLETOFT, R. J. The control of follicular wave development for self-appointed embryo transfer programs in cattle. Theriogenology, v. 57, n. 1, p. 53-72, 2002.

CALADO, A. M.; ROCHA, E.; COLAÇO, A; SOUSA, M. Stereologic characterization of bovine (Bos taurus) cumulus-oocyte complexes aspirated from small antral follicles during the diestrous phase. Biology of Reproduction, v. 65, p.1383-1391, 2001.

CARVALHO, J. B. P. Sincronizacão da ovulação com dispositivo intravaginal de progesterona (CIDR $R$ ) em novilhas Bos indicus, Bos indicus $x$ Bos taurus e Bos taurus. 2004. 122p. Tese (Doutorado) - Faculdade de Medicina Veterinária e Zootecnia, Universidade de São Paulo, São Paulo, 2004.

COLAZO, M. G.; KASTELIC, J. P.; MAPLETOFT, R. J. Effects of estradiol cypionate (ECP) on ovarian follicular dynamics, synchrony of ovulation, and fertility in CIDR-based, fixed-time AI programs in beef heifers. Theriogenology, v. 60, n. 5, p. 855-865, 2003. 
COLAZO, M. G.; MARTINEZ, M. F.; SMALL, J. A.; KASTELIC, J. P.; BURNLEY, C. A.; WARD, D. R.; MAPLETOFT, R. J. Effect of estradiol valerate on ovarian follicle dynamics and superovulatory response in progestin-treated cattle. Theriogenology, v. 63, n. 5, p. 14541468, 2005.

DISKIN, M. G.; AUSTIN, E. J.; ROCHE, J. F. Exogenous hormonal manipulation of ovarian activity in cattle. Domestic Animal Endocrinology, v.23, p. 211-228, 2002.

DODE, M. A. N. Avanços na maturação ovocitária em bovinos. Acta Scientiae Veterinariae. 34 (Supl1), p. 115-130, 2006.

DONALDSON, L. E. Superovulation of trial. Ausa International Inc. Tyler, Texas, 1992.

FIGUEIREDO, R. A.; BARROS, C. M.; PINHEIRO, O. L.; SOLER, J. M. Ovarian follicular dynamics in Nelore breed (Bos indicus) cattle. Theriogenology, v. 47, p. 1489-1505, 1997.

FORTUNE, J. E.; RIVERA, G. M.; YANG, M. Y. Follicular development: the role of the follicular microenvironment in selection of the dominant follicle. Animal Reproduction Science, v. 8283, p. 109-126, 2004.

GILCHRIST, R. B.; RITTER, L. J.; ARMSTRONG, D. T. Oocyte somatic cell interactions during follicle development in mammals. Animal Reproduction Science, v. 82-83, p. 431-446, 2004.

GINTHER, O. J.; WILTBANK, M. C.; FRICKE, P. M.; GIBBONS, J. R.; KOT, K. Selection of the dominat follicle in cattle. Biology of Reproduction, v. 55, p. 1187-1194, 1996.

GINTHER, O. J.; BEG, M. A.; BERGFELT, D. R.; DONADEU, F. X.; KOT, K. Follicle selection in monovular species. Biology of Reproduction, v. 65, p. 638-647, 2001.

GONÇALVES, P. B. D.; FIGUEIREDO, J. R.; FREITAS, V. J. F. Biotécnicas Aplicadas à Reprodução Animal. São Paulo: Varela, p. 195-226, 2002.

GONG, J. G.; CAMPBELL, B. K., BRAMLEY, T. A.; GUTIERREZ, C. G.; PETERS, A. R.; WeBB, R. Supression in the secretion of follicle stimulating hormone and luteinizing hormone, and ovarian follicle development in heifers continuously infused with a gonadotropina releasing hormone agonist. Biology of Reproduction, v. 55, p. 68-74, 1996.

GRRENWALD, G. S.; TERRANOVA, D. F. Follicular selection and its control. In: KNOBIL, E. \& NEILL, J. The physiology of reproduction. New York: Raven Press Ltda, p. $387-445,1988$.

GRUNERT, E.; GREGORY, R. M. Diagnóstico e terapêutica da infertilidade na vaca. $2^{a}$ ed. Porto Alegre: Editora Sulina, 1989, 174 p.

HAFEZ, E. S. E.; HAFEZ, B. Foliculogênese, maturação ovocitária e ovulação. In: HAFEZ, E. S. E.; HAFEZ, B. Reprodução Animal. 7a ed. São Paulo: Manole, p. 69-81, 2004.

JAINUDEEN, M. R \& HAFEZ, E. S. E. Ciclos reprodutivos: Bovinos e Bubalinos. In: HAFEZ, E. S. E.; HAFEZ, B. Reprodução Animal. 7a ed. São Paulo: Manole, Cap.11, p.159-171, 2004.

JUNQUEIRA, L. C.; CARNEIRO, J. C. Histologia Básica. 8a ed. Rio de Janeiro: Guanabara Koogan, 1995.

KAWAMATA, M. Relationships between the number of small follicles prior to superovulatory treatment and superovulatory response in Holstein cows. Journal of Veterinary Medicine Science, v. 56, p. 965-967, 1994. 
KNOPF, L.; KASTELIC, J. P., SCHALLENBERGER, E.; GINTHER, O. J. Ovarian follicular dynamics in heifers: test of two-wave hypothesis by ultrasonically monitoring individual follicles. Domestic Animal Endocrinology, v. 6, p. 111-119, 1989.

KOZICKI, L. E.; SEGUI, M. S.; FANTINI FILHO, J. C.; PRADO, F. R. A.; MATTÉ, F.; GLASER JR, P.; WEISS, R. R. A somatotrofina bovina (bST) e sua relação com o recrutamento folicular ovariano durante o ciclo estral de vacas. Archives of Veterinary Science, v. 10, n. 1, p. 35-44, 2005.

MACMILLAN, K. L.; THATCHER, W. W. Effects of an agonist of gonadotropin-releasing hormone on ovarian follicles in cattle. Biology of Reproduction, v.45, p.883-889, 1991.

MACMILLAN, K. L.; BURKE, C. R. Effects of oestrus cycle control on reproductive efficiency. Animal Reproduction Science, v. 42, p. 307-320, 1996.

MAPLETOFT, R. J.; STEWARD, K. B.; ADAMS, G. P. Recent advances in the superovulation in cattle. Reproduction Nutrition Development, v. 42, n. 6, p. 601-611, 2002.

MARIANI, A. C. B. Influência do dia estro em relação à FIV e a morfologia do corpo lúteo no dia da inovulação com os índices de prenhes em receptoras de embriões bovinos produzidos in vitro. 2009. 56 f. Mestrado (Ciência Animal Tropical) - Universidade Federal do Tocantins, Araguaína, 2009.

MARTINEZ, M. F.; ADAMS, G. P.; KASTELIC, J. P.; BERGFELT, D. R.; MAPLETOFT, R. J. Induction of follicular wave emergence for estrus synchronization and artificial insemination in heifers. Theriogenology, v.54, n. 5, p. 757-769, 2000.

MARTINS, C. M.; CASTRICINI, E. S. C.; SÁ FILHO, M. F.; GIMENES, L. U.; BARUSELLI, P. S. Dinâmica folicular em novilhas e vacas Nelore (Bos indicus) tratadas com dispositivo intravaginal de progesterona novo ou reutilizado associado ou não a progesterona injetável. Acta Scientiae Veterinarie, v. 33, p. 227, 2005. Suplemento1

MARTINS, F. S; SILVA, J. R. V.; RODRIGUES, A. P. R.; FIGUEIREDO, J. R. Fatores reguladores da foliculogênese em mamíferos. Revista Brasileira de Reprodução Animal, v.32, n.1, p.36-49, 2008.

MAX, M. C; ANDRADE, E. R; BASSO, A. C; FIGUEIREDO, J. R; SENEDA, M. M. Principais aspectos da manipulação de oócitos inclusos em folículos ovarianos pré-antrais. Revista Educação Continuada, v. 7, n. 1/3, p. 66-72, 2004.

MCNATTY, K. P.; HEATH, D. A.; LUNDY, T.; FIDLER, A. E.; QUIRKE, L. O'CONNELL, A.; SMITH, P.; GROOME, N.; TISDALL, D. J. Control of early ovarian follicular development. Journal of Reproduction and Fertility. Suppl, v. 54, p. 3-16, 1999.

MORAES, J. C. F.; SOUZA, C. J. H.; GONSALVES, P. B. D. Controle do estro e da ovulação em bovinos e ovinos. In: GONSALVES, P. B. D.; FIGUEIREDO, J. R.; FREITAS, V. J. F. Biotécnicas aplicadas à Reprodução Animal. São Paulo: Varela, 2002. cap. 3, p. 25-55.

MOREIRA, F.; DE LA SOTA, R. L.; DIAZ, T.; THATCHER, W. W. Effect of day of the estrous cycle at the initiation of a timed artificial insemination protocol on reproductive responses in dairy heifers. Journal of Animal Science, v.78, p. 1568-1576, 2000.

MORENO, D.; CUTAIA, L.; VILLATA, L.; ORTISI, F.; BO, G. A. Follicular wave emergence in beef cows treated with progesterone releasing devices, estradiol benzoate and progesterone. Theriogenology, v. 55, n. 1, p. 408, 2001. 
NASSER, L. F; ADAMS, G. P.; BO, G. A.; MAPLETOFT, R. J. Ovarian super stimulatory response relative to follicular wave emergence in heifers. Theriogenology, v. 40, p. 713-724, 1993.

NeVeS, M. M.; MARQUeS JR, A. P; SANTANA, C. V; LimA F. P. C.; ZAMBRANo, W. J. Características de ovários de fêmeas zebu (Bos taurus indicus), colhidos em abatedouros. Arquivo Brasileiro de Medicina Veterinária e Zootecnia, v. 54, n. 6, 2002.

NOGUEIRA, M. F. G.; BARROS, B. J.; TEIXEIRA, A. B.; TRINCA, L. A.; D'OCCHIO, M. J.; BARROS, C. M. Embryo recovery and pregnancy rates after the delay of ovulation and fixed time insemination in superstimulated beef cows. Theriogenology, v. 57, p. 1625-1634, 2002.

OKUDA, K.; MIYAMOTO, Y.; SKARZYNSKI, D. J. Regulation of endometrial prostaglandin $\mathrm{F}$ (2alpha) synthesis during luteolysis and early pregnancy in cattle. Domestic Animal Endocrinology, v. 23, p. 255-264, 2002.

PAVÃO, D. L. Avaliação da citotoxicidade e interferência da Ateleia glazioviana na interação do herpesvirus bovino tipo 1 (BoHV-1) com oócitos bovinos maturados in vitro. 2009. $60 \mathrm{f}$. Mestrado (Sanidade, Segurança Alimentar e Ambiental no Agronegócio) - Instituto Biológico, da Agência Paulista de Tecnologia do Agronegócio, São Paulo, 2009.

PIERSON, R. A.; GINTHER, O. J. Ultrasonography of the bovine ovary. Theriogenology, v. 21, p. 495-504, 1984.

PONTES, J. H; NONATO-JÚNIOR, I; SANCHES, B. V.; ERENO-JÚNIOR, J. C.; UVO, S; BARREIROS, T. R.; OLIVEIRA, J. A.; HASLER, J. F.; SENEDA, M. M. Comparison of embryo yield and pregnancy rate between in vivo and in vitro methods in the same Nelore (Bos indicus) donor cows. Theriogenology, v. 71, p. 690-697, 2009.

PURSLEY, J. R.; MEE, M. O.; WILTBANK, M. C. Synchronization of ovulation in dairy cows using PGF2alpha and GnRH. Theriogenology, v. 44, n. 7, p. 915-923, 1995.

REINCHENBACH, H. Transferência e criopreservação de embriões bovinos. In: GONÇALVES, P. B. D.; FIGUEIREDO, J. R.; FREITAS, V. J. F. Biotécnicas Aplicadas à Reprodução Animal. São Paulo: Varela, p. 127-177, 2002.

RIVERA, G. Regulación neuroendocrina de la función ovárica. In: PALMA, G. A.; BREM, G. Transferencia de embriones y biotecnología de la reproducción en la especie bovina. p. 4346, 1993.

RUBIN, K. C. P. Particularidades reprodutivas da raça Nelore na produção in vitro de embriões (PIVE). 2006. 64 f. Especialização (Ciência Animal) - Universidade Estadual de Londrina, Paraná, 2006.

RUMPF, R.; BEM, D. E.; PEIXER, M. A. S.; SOUZA, R. V. Manual de transferência e micromanipulação de embriões nas espécies bovina e eqüina. Brasília: EMBRAPA - Recursos Genéticos e Biotecnologia, 2000. p. 71-103.

SÁ FILHO, M. F.; GIMENES, L. U.; TORRES-JÚNIOR, J. R. S; CARVALHO, N. A. T.; KRAMER, M. P. S.; FARIA, M. H.; BARUSELLI, P. S. Emergência folicular conforme a dose e o momento de aplicação do Benzoato de Estradiol e de sua associação com progesterona injetável em vacas Nelore (Bos indicus) tratadas com dispositivo intravaginal de progesterona. IRAC, 2005. 
SÁ FILHO, M. F.; ReIS, E. L.; AYReS, H.; Gimenes, A. A. C.; PERES, A. A. C.; CARVAlho, C. A. B.; CARVALHO, J. B.; ARAÚJO, C. A. S. C.; BARUSELLI, P. S. Effect of oestradiol valerate or benzoate on induction of a new follicular wave emergence in Bos indicus cows and heifers treated with norgestomet auricular implant. Journal of Reproduction, Fertility and Development, v. 18, p. 289, 2006.

SERAPIÃO, R. V. Desenvolvimento de embriões bovinos produzidos in vitro cultivados em meio livre de soro. Revista Brasileira de Reprodução Animal, v. 32, n. 1, p. 9-15, 2008.

SILCOX, R. W.; POWELL, K. L.; KISER, T. E. Ability of dominant follicles to respond to exogenous GnRH administration is dependent on their stage of development. Journal of Animal Science, v.71, suppl. 1, p. 219, 1993.

SPICER, L. J.; ECHTERNKAMP, S. E. Ovarian follicular growth, function and turnover in cattle: a review. Journal of Animal Science, v. 62, p. 428-451, 1986.

THIBIER, M. New records in the numbers of both in vivo-derived and in vitro-produced bovine embryos around the world in 2006. International Embryo Transfer Society Newsletter, v. 25, p. $15-20,2007$.

YAAKUB, H.; O'CALLAGHAN, D.; BOLAND, M. P. Effect of type and quantity of concentrates on superovulation and embryo yield in beef heifers. Theriogenology, v. 51, p. 1259-1266, 1999.

WEBB, R.; NICHOLAS, B.; GONG, J. G.; CAMPBELL, B. K.; GUTIERREZ, C. G.; GARVERICK, H. A.; ARMSTRONG, D. G. Mechanisms regulating follicular development and selection of the dominant follicle. Reproduction Suppl, v. 61, p. 71-90, 2003. 